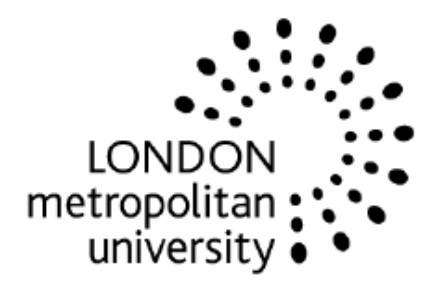

Centre for International Capital Markets

Discussion Papers

ISSN 1749-3412

A General Equilibrium and Preference Free Model for Pricing Options under

Transformed Gamma Distribution

Luiz Vitiello, Ser-Huang Poon

No 2008-13 


\title{
A general equilibrium and preference free model for pricing options under transformed gamma distribution
}

\author{
Luiz Vitiello and Ser-Huang Poon*
}

September 29, 2008

\footnotetext{
${ }^{*}$ Corresponding author. Ser-Huang Poon is at Manchester Business School, Crawford House, University of Manchester, Oxford Road, Manchester M13 9PL, UK. Tel: +44 161275 0431, Fax: +44 161275 4023, Email: ser-huang.poon@mbs.ac.uk. Luiz Vitiello is at London Metropolitan Business School, London Metropolitan University. We are grateful to Michael Brennan, Richard Stapleton and Konstantinos Vonatsos for valuable insights. We would like to thank the seminar participants at Singapore Management University for helpful comments.
} 


\title{
A general equilibrium and preference free model for pricing options under transformed gamma distribution
}

\begin{abstract}
The gamma class of distributions encompasses several important distributions either as special or limiting cases, or through simple transformations. Here we derived closed form and preference free European option pricing formulae for a variety of (transformed) gamma distributions under the general equilibrium RNVR framework. The gamma class of distributions is used historically in hydrology for modelling natural events. Our models can be used to price derivatives associated with these natural phenomena, which will help to encourage greater risk sharing through financial securitization. Our pricing formulae are theoretically sound even if the underlings and the derivative instruments are not (frequently) traded.
\end{abstract}

Keywords: Risk Neutral Valuation Relationship, General Equilibrium framework, transformed gamma distribution, Weather Derivatives.

JEL classification: G12, G13, G22. 


\section{A general equilibrium and preference free model for pricing options under transformed gamma distribution}

\section{Introduction}

Hitherto, studies of contingent claims under the general equilibrium framework have relied heavily on the assumption of normal and lognormal distributions to produce option pricing formulae (e.g. Black and Scholes (1973), Rubinstein (1976, 1983), Brennan (1979) and Camara (2003)). It is well known that many asset distributions are not Gaussian. In the recent years, option exchanges have started trading contracts that are written on exotic underlyings such as volatility and weather, whose distributions are clearly not $(\log )$ normal. It is our objective here to expand the underlying distributions in option pricing theories to beyond Gaussian class of distributions to gamma class of distributions which is one of the most important distributions in hydrology studies.

In this paper, we establish the risk neutral valuation relationships (RNVR) and derive European option pricing formulae for the gamma class of distributions. The gamma distribution can deliver several other distributions, either as a special or limiting case, or through a simple transformation. Among others, distributions that are associated with the gamma distribution include the exponential distribution, the normal distribution and the family of extreme-value distributions, viz. Pareto, Weibull and Gumbel distributions. The gamma class of distributions, in general, has been used in hydrology for modelling natural extreme events such as flood, rain and wind (e.g. See Stern and Coe (1984), Loukas, Vasiliades, Dalezios and Domenikiotis (2001), Yue, Ourada, and Bobee (2001) and Sharda and Das (2005)). 
Option pricing formulae that are based on the gamma class of distributions can help to encourage the securitization of financial costs related to these natural phenomena as part of a wider risk sharing mechanism.

In our models, we assume that the underlying has a transformed gamma distribution. Different risk preferences are obtained depending on the wealth distributions. The pricing kernel and asset specific pricing kernel proposed are obtained from the general equilibrium argument following Brennan (1979). In particular, this paper follows closely the approach used in Camara (2003) for deriving option pricing formulae based on transformed normal distributions. For each of the option pricing formulae that we derived, we establish, in the first instance, the existence of a RNVR between the underlying and the option price. Option prices derived under this RNVR framework are preference free. That is, the investor's risk aversion parameter does not appear in the option pricing formulae. Also, the market does not have to be dynamically complete. This allows us to produce prices for derivatives even in cases where the derivatives and the underlyings are illiquid or not traded. This is the key feature that motivates Brennan (1979) who notes that costs which will be paid only in the event of bankruptcy, certain kinds of tax liability and the opportunity of a firm to make profitable investments in the future are examples of contingent claims that are not traded.

The gamma class of distributions has been used in option pricing applications before (e.g. Heston (1993), Gerber and Shiu (1994), Lane and Movchan (1999), Savickas (2002), Schroder (2004)). But the model presented here differs from these for at least one of the following reasons: (i) It is based on a monotonic transformation of the gamma distributions, the transformed gamma, which includes several other well known distributions; (ii) It explicitly shows, by construction, that it is possible 
to achieve a risk neutral valuation relationship in an economy with transformed gamma asset distributions. Risk neutrality is attained by equilibrium arguments rather then by assuming that investors are risk neutral; (iii) It shows the exact link between the primitves of the economy and the asset specific pricing kernel.

The remainder of this paper is organized as follows: in Section II the economy is introduced. Section III presents the assumptions on distributions and preferences. Section IV defines the pricing kernel and the asset specific pricing kernel. In Section $\mathrm{V}$, the basic framework for pricing European-style options is introduced and several new pricing formulae, all related to the gamma class of distributions, are derived. Section VI concludes.

\section{The Basic Set Up}

In this framework, the market is complete with a Pareto-optimal allocation. The representative investor ${ }^{1}$ who maximizes his expected utility of end of period wealth, $W_{T}$, according to

$$
\begin{gathered}
\max _{a} E^{P}\left[U\left(W_{T}\right)\right] \\
W_{T}=W \exp (r T)+\sum_{j=1}^{N} a_{j}\left(S_{T, j}-F_{j}\right),
\end{gathered}
$$

where $W$ is the initial wealth, $S_{T, j}$ is the price of the risky asset $j$ at time $T, F_{j}$ is the forward price, $r$ is the risk free rate, $a_{j}$ is the number of units of the risky asset $j$ purchased, and the superscript $P$ of $E(\cdot)$ means that the expectation is taken with respect to the actual probability measure.

\footnotetext{
${ }^{1}$ The existence of a representative agent is ensured by a Pareto-optimum market. See (Huang and Litzenberger (1988), Ch. 5).
} 
Considering a risk-averse investor (i.e. $\left.U^{\prime \prime}\left(W_{T}\right)<0\right),{ }^{2}$ the problem is solved by satisfying the first order condition for a maximum. ${ }^{3}$ Dropping the subscript $j$, the initial price of any asset in this economy is given by:

$$
\begin{aligned}
F & =\frac{E^{P}\left[U^{\prime}\left(W_{T}\right) S_{T}\right]}{E^{P}\left[U^{\prime}\left(W_{T}\right)\right]} \\
& =E^{P}\left[\phi\left(W_{T}\right) S_{T}\right],
\end{aligned}
$$

where

$$
\phi\left(W_{T}\right)=\frac{U^{\prime}\left(W_{T}\right)}{E^{P}\left[U^{\prime}\left(W_{T}\right)\right]}
$$

is defined as the pricing kernel. ${ }^{4}$ Note that, for a complete market set up, this pricing kernel is unique.

Conditioning $\phi\left(W_{T}\right)$ in equation (4) with respect to the risky asset leads to the asset specific pricing kernel,

$$
\psi\left(S_{T}\right)=E^{P}\left[\phi\left(W_{T}\right) \mid S_{T}\right]
$$

which is also known as the conditional expected relative marginal utility function. Equation (5) is the projection of the pricing kernel, given by equation (4), onto the space of $S_{T}$.

\footnotetext{
${ }^{2} \mathrm{~A}$ risk-averse agent is the one who "starting from a position of certainty, is unwilling to take a bet which is actuarially fair (a fortiori, he is unwilling to take a bet which is actuarially unfair to him)" (Arrow (1974), p. 90).

${ }^{3}$ For simplicity, drop the subscript $j$. The first order condition for a maximum is given by $E^{P}\left[U^{\prime}\left(W_{T}\right)\left(S_{T}-F\right)\right]=0$, where $U^{\prime}\left(W_{T}\right)$ is the marginal utility function defined over the terminal wealth. Solving for $F$ yields equation (3). A detailed derivation is presented in chapter 5 of Huang and Litzenberger (1988) and chapter 1 of Poon and Stapleton (2005).

${ }^{4}$ Brennan (1979) calls it "the relative marginal utility of wealth of the representative investor".
} 
Thus, equation (3) can be rewritten as

$$
F=E^{P}\left[\psi\left(S_{T}\right) S_{T}\right]
$$

which is known as the basic valuation equation, and can be used to price the risky asset $S_{T}$ and any contracts or derivative securities written on $S_{T}$.

\section{Distributions and Preferences}

The distributional form of both the wealth and the price of the risky asset play a fundamental role in the pricing framework described in the previous section. In this section we introduce the distributional assumptions that underlie this study. Specifically, we present the gamma distribution and define the transformed gamma distribution.

\section{A The Gamma Distribution}

The gamma density is defined as

$$
f(x)=\frac{a^{p}}{\Gamma(p)} x^{p-1} e^{-a x},
$$

where $p, a>0,0 \leq x<\infty$, and $\Gamma(\cdot)$ is the gamma function

$$
\Gamma(p)=\int_{0}^{\infty} z^{p-1} e^{-z} d z .
$$

Note that, similar to the lognormal distribution, the gamma distribution is restricted to positive values of $x$ only. The probability of $x$ being less then $X$ is given by the gamma probability distribution function $G(X, p) \equiv \Gamma(X, p) / \Gamma(p)$, where 
$\Gamma(\cdot, \cdot)$ is the incomplete gamma function

$$
\Gamma(X, p)=\int_{0}^{X} x^{p-1} e^{-x} d x
$$

The gamma distribution is commonly used in hydrology research and in the analysis of survival data. For $p=1$, equation (7) becomes an exponential density. If $p$ is an integer, equation (7) becomes an Erlang distribution. For $p=v / 2$ and $a=1 / 2$, equation (7) becomes a chi-squared distribution with $v$ degrees of freedom. As $p \rightarrow \infty$ the gamma distribution converges to the normal distribution.

The shape of the gamma distribution for $a=1$ and for different values of $p$ is presented in Figure 1. It is possible to see that for small values of $p$ the distribution is highly skewed, but as $p$ increases the distribution becomes more symmetrical. Two special cases presented in Figure 1 are $p=1$ (the exponential distribution) and $p$ integer (the Erlang distribution). Note that the exponential distribution is also a special case of the Erlang distribution.

Define $h(z)$ to be some transformation of $z$. If $x$ in $h(z)=a x$ has a gamma density according to equation $(7)$ and $h(\cdot)$ is a monotonic differentiable function then the density of $z$ is given by

$$
f(z)=\frac{1}{\Gamma(p)}\left|h^{\prime}(z)\right| h(z)^{p-1} e^{-h(z)}
$$

where $h^{\prime}(z)$ is the first derivative of $h(z)$. Here $h(z)$ is gamma distributed and $f(z)$ is a transformed gamma density.

For $p=1$, the transformed gamma distribution nests several important distributions. For example, if $h(z)=\exp (z)$, then equation (9) becomes the standard Gumbel density. ${ }^{5}$ The Gumbel distribution has been used to model floods, earth-

\footnotetext{
${ }^{5}$ The Gumbel is Type I of the three classes of extreme value distributions. It corresponds to
} 


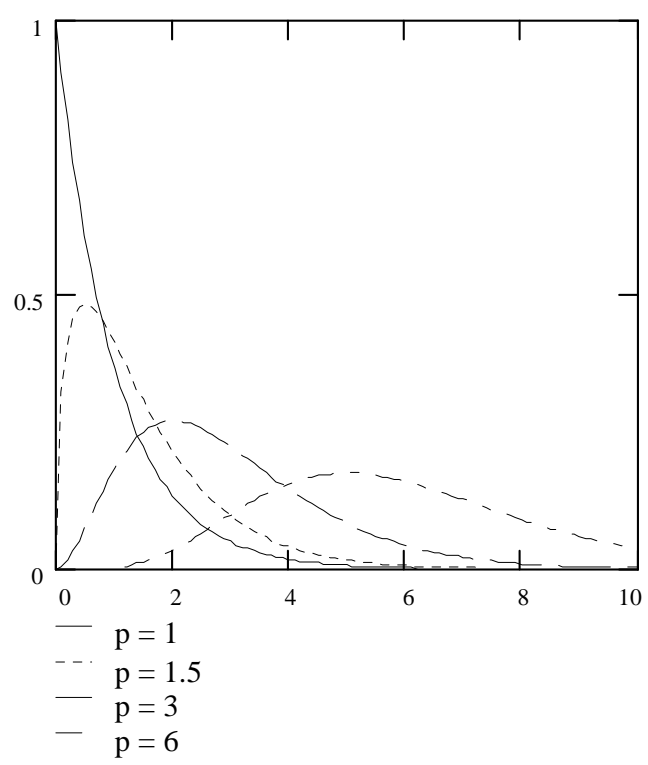

Figure 1: The gamma density function.

quakes, athletic fastest records and maximal such as the hottest day, the wettest month etc. A typical shape of the Gumbel distribution is presented in Figure 2.

If $p=1$ and $h(z)=z^{b}$, then equation (9) becomes a Weibull distribution. The shape of the Weibull distribution for different values of $b$ is presented in Figure 3. Note that when $b=1$ the Weibull distribution collapses into the exponential distribution.

Also for $p=1$, amongst other possibilities, if $h(z)=z^{2} / 2$, equation (9) becomes a Rayleigh distribution. If $h(z)=b \ln (z)$, equation (9) becomes a Pareto distribution.

logarithmic transformations of Type II (Frechet) and Type III (Weibull) extreme value distributions. 


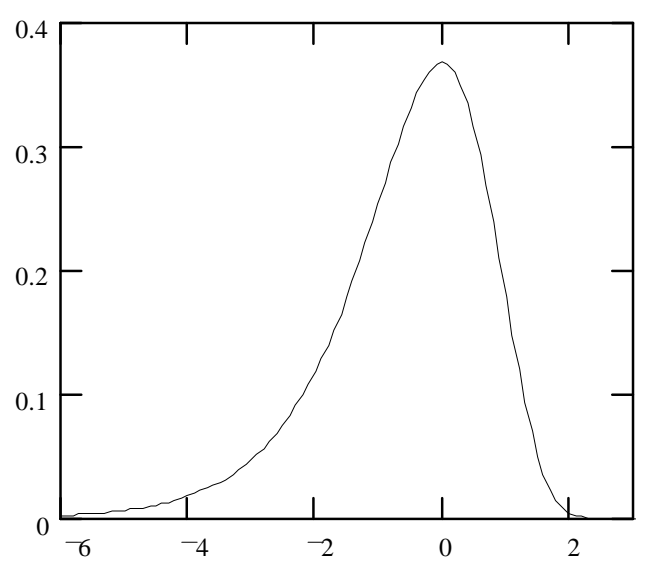

Figure 2: The Gumbel density function.

\section{B The Gamma Bivariate Density}

In contrast to the normal distribution, which has only one specification for the bivariate density function, the gamma distribution has several depending on the method used to construct these bivariate gamma distributions. ${ }^{6}$ In this paper, it is assumed that the joint distribution is represented by the Mckay (1934) bivariate gamma density presented in the following definition. ${ }^{7}$

Definition 1 (The bivariate gamma density) Let the random variables $x$ and $y$ have the joint density

$$
f(x, y ; p, q, a)=\frac{a^{p+q}}{\Gamma(p) \Gamma(q)} x^{p-1}(y-x)^{q-1} e^{-a y},
$$

\footnotetext{
${ }^{6}$ See Mardia (1970) and Hutchinson and Lai (1990).

${ }^{7}$ The Mckay bivariate gamma density is chosen because it has a simple representation, involving only one additional parameter.
} 


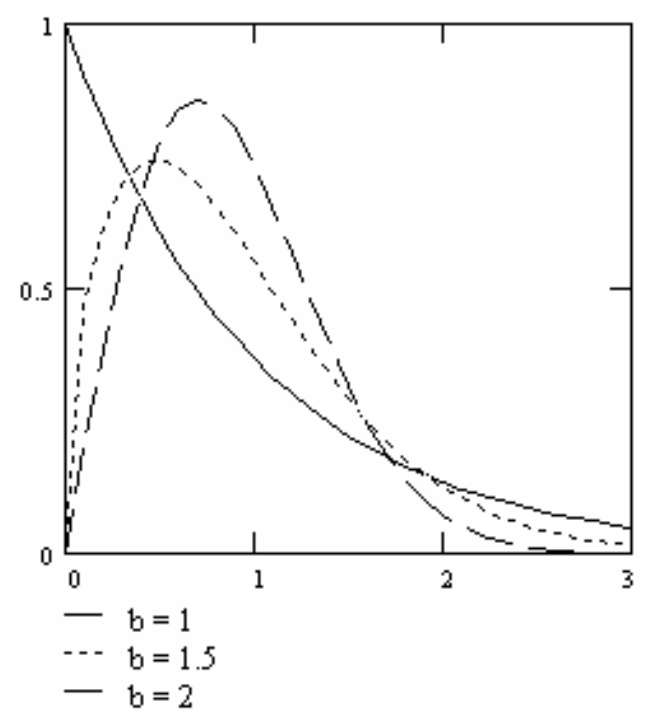

Figure 3: The Weibull density function.

where

$$
\begin{gathered}
a^{2}=\frac{p}{\sigma_{x, y}}, \\
\rho_{x, y}=\sqrt{\frac{p}{p+q}},
\end{gathered}
$$

for $y>x>0$, and $a, p, q>0$.

Then $x$ and $y$ have gamma marginal densities given respectively by:

$$
\begin{gathered}
f(x ; p, a)=\frac{a^{p}}{\Gamma(p)} x^{p-1} e^{-a x}, \\
f(y ; p+q, a)=\frac{a^{p+q}}{\Gamma(p+q)} y^{p+q-1} e^{-a y} .
\end{gathered}
$$

Since $a, p, q>0$, the covariance, $\sigma_{x, y}$, and the correlation, $\rho_{x, y}$, are strictly positive. Covariance and correlation tend to zero only if $p$ also tends to zero. Both 
marginal densities $f(x)$ and $f(y)$ are gamma densities but it is not always possible to have both densities belonging to the same type. For example, since $p$ and $q$ are both strictly positive, $p$ and $p+q$ cannot be both equal to 1 . Hence, $x$ and $y$ cannot be both exponential. In general, all transformations that require $p=1$ will lead to $x$ and $y$ having different marginal densities.

\section{Risky Asset and Wealth Distribution}

The asset specific pricing kernel is derived from the joint distribution of wealth and the underlying. From the bivariate gamma density in Definition 1 and the univariate transformed gamma density from (9), we can now specify the distributional assumptions for wealth and the underlying.

Definition 2 (Underlying and wealth distributions) The terminal value of the underlying, $S_{T}$, and the terminal wealth, $W_{T}$, have a rescaled marginal gamma distribution given respectively by

$$
\begin{gathered}
h\left(S_{T}\right)=\mu+\sigma x \\
h_{W}\left(W_{T}\right)=\mu_{W}+\sigma_{W} y
\end{gathered}
$$

where $h(\cdot)$ is a monotonic differentiable function, $x$ and $y$ have a joint gamma density according to equation (10) with gamma marginal densities according to equations (12) and (13) respectively.

Since the gamma distribution encompasses the normal distribution as a limiting case, the distribution assumption of wealth and risky asset provided in Definition 1 are in line with the assumptions adopted by Brennan (1979). For instance, 
one could have a normally distributed wealth and a gamma distributed underlying. Definition 1 provides a lot of flexibility for choosing the marginal distribution of $W_{T}$ and $S_{T}$ from a range of gamma or transformed gamma distributions.

\section{The Pricing Kernel}

Given Definition 2, it is now possible to specify the pricing kernel in equation (4) and the asset specific pricing kernel in equation (5), which is done in the following propositions.

Proposition 3 (The pricing kernel) Assume a representative investor with marginal utility function given by

$$
U^{\prime}\left(W_{T}\right)=\exp \left[\gamma h_{W}\left(W_{T}\right)\right]
$$

where $\gamma$ is a constant preference parameter, and $h_{W}\left(W_{T}\right)$ has a rescaled gamma distribution according to equation (15). Then, the pricing kernel is given by

$$
\phi\left(W_{T}\right)=a^{-(p+q)}\left(a-\gamma \sigma_{W_{T}}\right)^{p+q} \exp \left[-\gamma \mu_{W_{T}}+\gamma h_{W}\left(W_{T}\right)\right] .
$$

Proof. See Appendix.

In Proposition 3, the representation for the marginal utility function is very convenient, as the investor's preference is controlled by the functional form of $h_{W}\left(W_{T}\right)$. Thus, if $h_{W}\left(W_{T}\right)=W_{T}$, the representative investor has an marginal exponential utility function characterized by constant absolute risk aversion (CARA). If $h_{W}\left(W_{T}\right)=\ln \left(W_{T}\right)$, the representative investor has a marginal power utility function with constant relative risk aversion (CRRA). 
Proposition 4 (The asset specific pricing kernel) Assume that Proposition 3 holds. Assume also that the joint distribution of the terminal wealth and the terminal value of the underlying is given by Definition 2. Then, the asset specific pricing kernel is given by

$$
\psi\left(S_{T}\right)=a^{-p}\left(a-\gamma \sigma_{W_{T}}\right)^{p} \exp \left[\gamma \sigma_{W_{T}}\left(\frac{h\left(S_{T}\right)-\mu}{\sigma}\right)\right]
$$

Proof. See Appendix.

An interesting aspect of the above proposition is that any function $h_{W}\left(W_{T}\right)$ that satisfies Proposition 3 also satisfies the requirements of Proposition 4 and, consequently, delivers the same asset specific pricing kernel. That is, the functional form of $h_{W}\left(W_{T}\right)$ does not change the functional form of the asset specific pricing kernel in equation (18).

Corollary 5 The functional form of the risk adjusted density of $S_{T}$, which is given by the product of the actual density of $S_{T}$ in (14) and the asset specific pricing kernel in (18) is a transformed gamma density with location $\mu$ and scale $\left(a-\gamma \sigma_{W}\right) / \sigma$.

Proof. It follows directly from the definition of the asset specific pricing kernel, $\psi\left(S_{T}\right)$, and the transformed density of $S_{T}, f\left(S_{T}\right)$, given in Definition 2.

This corollary shows that in the transformed gamma framework, only the scale parameter is affected by the preference parameter. This is in sharp contrast to the transformed normal case of Camara (2003) where only the location parameter is affected by preference. 


\section{V option pricing Formulae}

In order to obtain preference free option pricing models, it is necessary to eliminate from the formula the parameter that is related to the investor's preference. This is achieved by substituting the asset specific pricing kernel in (18) into equation (6) to yield

$$
F=\int S_{T} \psi\left(S_{T}\right) f\left(S_{T}\right) d S_{T}
$$

If the above expectation has a closed form solution, it may be possible to isolate the risk aversion parameter and replace it with observable parameters, such as securities price. Assuming that this is possible, take a call option with a payoff $\max \left(S_{T}-K, 0\right)$ as an example, where $K$ is the option strike price, the price of this call option is

$$
\begin{aligned}
C e^{r T} & =E^{P}\left[\max \left(S_{T}-K, 0\right) \psi\left(S_{T}\right)\right] \\
& =\int \max \left(S_{T}-K, 0\right) \psi\left(S_{T}\right) f\left(S_{T}\right) d S_{T} .
\end{aligned}
$$

where the density $f\left(S_{T}\right)$ involves preference parameters. It is then possible to substitute these preference parameters by prices to obtain a preference free option pricing formulae

$$
\begin{aligned}
C e^{r T} & =\int \max \left(S_{T}-K, 0\right) f^{*}\left(S_{T}\right) d S_{T} \\
& =E^{Q}\left[\max \left(S_{T}-K, 0\right)\right]
\end{aligned}
$$

where $f^{*}\left(S_{T}\right)$ is the risk-neutral density and the superscript $Q$ of $E(\cdot)$ means that the expectation is taken with respect to the risk-neutral probability measure. That is, the option price can be regarded as a martingale with respect to $Q$. 
Given the above discussion, it is clear that the possibility of obtaining a preference free option pricing formula is strongly related to the functional form of $\psi\left(S_{T}\right)$ and $f\left(S_{T}\right)$. We show, in the examples below, that the application of Definition 2 and Proposition 4 can lead to preference free option pricing formulae. ${ }^{8}$ The examples are for European-style call options only, but it is possible to obtain European-style put options by using similar arguments.

\section{A Log gamma}

Example 6 (Log gamma option pricing formula) Assume that the terminal wealth and the terminal value of the underlying are given, respectively, by $h\left(W_{T}\right)=\ln \left(W_{T}\right)$ and $h\left(S_{T}\right)=\ln \left(S_{T}\right)$. In this case, investors present CRRA. Using equations (17) and (18), the pricing kernel and the asset specific pricing kernel are given respectively by

$$
\begin{gathered}
\phi\left(W_{T}\right)=a^{-(p+q)}\left(a-\gamma \sigma_{W_{T}}\right)^{p+q} \exp \left[-\gamma \mu_{W_{T}}+\gamma \ln \left(W_{T}\right)\right], \\
\psi\left(S_{T}\right)=a^{-p}\left(a-\gamma \sigma_{W_{T}}\right)^{p} \exp \left[\gamma \sigma_{W_{T}}\left(\frac{\ln \left(S_{T}\right)-\mu}{\sigma}\right)\right] .
\end{gathered}
$$

Substitute the asset specific pricing kernel in equation (22) and the density function of the terminal value of the underlying from Definition 2 into equation (19) yields

$$
\left(a-\gamma \sigma_{W_{T}}\right)=\frac{\left(F e^{-\mu}\right)^{1 / p} \sigma}{\left(F e^{-\mu}\right)^{1 / p}-1} .
$$

${ }^{8}$ The proofs to the first example are provided in the Appendix. The results presented in the others examples can be obtained similarly. 
Rearranging this formula and substituting it into equation (20) gives the call option pricing formula

$$
C=F e^{-r T}\left[1-G\left(d_{1}, p\right)\right]-K e^{-r T}\left[1-G\left(d_{2}, p\right)\right],
$$

where $G(\cdot, \cdot)$ is the gamma probability distribution function and

$$
\begin{gathered}
d_{1}=\frac{(\ln (K)-\mu)}{\left(F e^{-\mu}\right)^{1 / p}-1}, \\
d_{2}=d_{1}+(\ln (K)-\mu) .
\end{gathered}
$$

for $\ln (K)>\mu, F e^{-\mu}>1$, and $p>0$.

A special case of the asset specific pricing kernel and the equilibrium relationship presented above, i.e. equations (22) and (23) respectively, is the model developed by Heston (1993). Heston assumes an asset specific pricing kernel of the form $S_{T}^{-\gamma}$, which clearly has less parameters than equation (22). In fact, Heston's asset specific pricing kernel and equation (22) could be the same only when $p=0$, which contradicts the definition of the gamma distribution. As it can be seen in equation (7), the gamma distribution requires $p>0$. The difference between Heston's asset specific pricing kernel and equation (22) is due to the fact that the asset specific pricing kernel used by Heston is arbitrarily chosen and all the other distributional parameters are simply ignored.$^{9}$

\footnotetext{
${ }^{9}$ As (Franke, Huang and Stapleton (2004), p. 1) point out: "Heston's set of preferenceparameter-free valuation relationship is somewhat difficult to apply. Unless we have knowledge of all other parameters of the pricing kernel, apart from the missing parameter, options cannot be priced using a preference-parameter-free valuation relationship".
} 


\section{B Log chi-square}

Example 7 (Log chi-squared option pricing formula) It is possible to obtain another special case of equation (24) by assuming that the logarithm of the value of the underlying has a chi-squared distribution. All the other assumptions are the same. From Definition 2, setting $p=v / 2$ and $h\left(S_{T}\right)=\ln \left(S_{T}\right) / 2$ yields the chi-squared density

$$
f\left(S_{T}\right)=\frac{a^{v / 2}}{\Gamma(v / 2)} \frac{1}{2^{v / 2} \sigma S_{T}}\left(\frac{\ln \left(S_{T}\right)-\mu}{\sigma}\right)^{v / 2-1} \exp \left(-a \frac{\ln \left(S_{T}\right)-\mu}{2 \sigma}\right),
$$

for $\left[\ln \left(S_{T}\right)-\mu\right]>0$.

In this specific case, since the scale parameter, $\sigma$, does not appear in equation (24), it is possible to obtain the log chi-squared pricing kernel, asset specific pricing kernel and the option pricing formula directly from equation (24) by setting $p=v / 2$. In this case, the asset specific pricing kernel and the equilibrium relationship are given, respectively, by

$$
\begin{gathered}
\psi\left(S_{T}\right)=a^{-v / 2}\left(a-\gamma \sigma_{W_{T}}\right)^{v / 2} \exp \left[\gamma \sigma_{W_{T}}\left(\frac{\ln \left(S_{T}\right)-\mu}{2 \sigma}\right)\right] . \\
F=\frac{e^{\mu}\left(a-\gamma \sigma_{W_{T}}\right)^{v / 2}}{\left(a-\gamma \sigma_{W_{T}}-2 \sigma\right)^{v / 2}}
\end{gathered}
$$

and the option pricing formula is given by

$$
C=F e^{-r T}\left[1-G\left(d_{1}, v / 2\right)\right]-K e^{-r T}\left[1-G\left(d_{2}, v / 2\right)\right],
$$

where $G(\cdot, \cdot)$ is the gamma probability distribution function and

$$
\begin{gathered}
d_{1}=\frac{(\ln (K)-\mu)}{\left(F e^{-\mu}\right)^{2 / v}-1}, \\
d_{2}=d_{1}+(\ln (K)-\mu)
\end{gathered}
$$

for $\ln (K)>\mu$ and $[F \exp (-\mu)]^{2 / v}>1$. 


\section{Weibull}

Example 8 (Weibull option pricing formula) Assume that terminal wealth and the terminal value of the underlying have a rescaled gamma distribution according to Definition 2. Assume also that wealth in (15) is given by $h_{W}\left(W_{T}\right)=W_{T}$ and the terminal value of the underlying in (14) is given by $h\left(S_{T}\right)=\sigma\left[\left(S_{T}-\mu\right) / \sigma\right]^{b}+\mu$, with $p=1$. That is, $W_{T}$ has a gamma density and $S_{T}$ has a Weibull density given by

$$
f\left(S_{T}\right)=\frac{a b}{\sigma}\left(\frac{S_{T}-\mu}{\sigma}\right)^{b-1} \exp \left[-a\left(\frac{S_{T}-\mu}{\sigma}\right)^{b}\right],
$$

for $S_{T}>\mu$ and $b>0$.

According to these assumptions, investors present CARA, and the asset specific pricing kernel is given by

$$
\psi\left(S_{T}\right)=\left(1-a^{-1} \gamma \sigma_{W_{T}}\right) \exp \left[\gamma \sigma_{W_{T}}\left(\frac{S_{T}-\mu}{\sigma}\right)^{b}\right] .
$$

Substituting the asset specific pricing kernel in equation (30) and the density function of $S_{T}$ in equation (29) into the asset pricing relationship in equation (19) yields

$$
F=\frac{\sigma \Gamma(1 / b+1)}{\left(a-\gamma \sigma_{W_{T}}\right)^{1 / b}}+\mu
$$

where $\Gamma(\cdot)$ is the gamma function as defined before. Solving equation (20) and using the above relationship yields the following call option pricing formula

$$
C=(F-\mu) e^{-r T}\left[1-G\left(d_{1}, 1 / b+1\right)\right]-(K-\mu) e^{-\left(d_{1}+r T\right)}
$$

where $G(\cdot, \cdot)$ is the gamma probability distribution function and

$$
d_{1}=\left[\left(\frac{K-\mu}{F-\mu}\right) \Gamma\left(\frac{1}{b}+1\right)\right]^{b},
$$

for $F, K>\mu$ and $b>0$. 


\section{Log Gumbel}

Example 9 (Log Gumbel option pricing formula) Assume that terminal wealth and the terminal value of the underlying have a rescaled gamma distribution according to Definition 2. Assume also that $h_{W}\left(W_{T}\right)=W_{T}$, and $h\left(S_{T}\right)=\sigma \exp \left[\left(\ln \left(S_{T}\right)-\mu\right) / \sigma\right]+$ $\mu$, and $p=1$. In this case, $W_{T}$ has a gamma density and $S_{T}$ has a Gumbel distribution with density

$$
f\left(S_{T}\right)=\frac{a}{\sigma S_{T}} \exp \left[\frac{\ln \left(S_{T}\right)-\mu}{\sigma}\right] \exp \left[-a e^{\left[\ln \left(S_{T}\right)-\mu\right] / \sigma}\right] .
$$

The asset specific pricing kernel is

$$
\psi\left(S_{T}\right)=\left(1-a^{-1} \gamma \sigma_{W_{T}}\right) \exp \left[\gamma \sigma_{W_{T}} e^{\left[\ln \left(S_{T}\right)-\mu\right] / \sigma}\right] .
$$

The equilibrium relationship is given by

$$
F=\frac{e^{\mu} \Gamma(1+\sigma)}{\left(a-\gamma \sigma_{W_{T}}\right)^{\sigma}}
$$

and the option pricing formula is

$$
C=F e^{-r T}\left[1-G\left(d_{1}, 1+\sigma\right)\right]-K e^{-\left(d_{1}+r T\right)}
$$

where $G(\cdot, \cdot)$ is the gamma probability distribution function and

$$
d_{1}=\left(\frac{K}{F} \Gamma(1+\sigma)\right)^{1 / \sigma}
$$

\section{Conclusion}

This paper presents a general equilibrium framework for pricing European options written on underlying that has a transformed gamma distribution. This framework, 
which guarantees that the resulting pricing model is preference free, allows us to obtain new set of European option pricing formulae even in cases where the derivatives are illiquid, or where the underlyings are illiquid or not traded. Unlike previous applications of gamma distribution in option pricing, this paper established a clear link between the real and the risk neutral distributions, and provided a formal proof for the existence of a risk neutral valuation relationship between option price and the underlying asset. Our paper extends the distributional and preference assumptions of the gamma option pricing model developed by Heston (1993), and can be seen as a parallel development complementing the work of Camara (2003) in option pricing based on transformed normal distributions.

In our model, the terminal wealth and the terminal value of the underlying do not have to have same distribution provided that they both belong to the transformed gamma distribution of which the normal distribution is a limiting case. The gamma distribution can produce several other important distributions as special or limiting cases or through simple transformations. We have demonstrated, in this paper, how risk neutral European option pricing formulae can be derived for (transformed) gamma distributions with a few examples. The same steps can be used to derive European option pricing formulae for other gamma class of distributions and transformed gamma distributions. Given the flexibility of the gamma distribution, our paper significantly expands the set of underlying distributions embedded in current option pricing theories. It has already been suggested (e.g. Savickas (2002), Lane and Movchan (1999)) that the gamma class of distributions could, in some cases, produce a better fit to the empirical observations than the Gaussian class of distributions, and that it will play a key role in the pricing of derivatives written on natural events. Our research and results are timely, and will help to encourage 
greater use of financial securitization in risk sharing. 


\section{Appendix}

Proof. (Equation 17 on the pricing kernel) Given the marginal utility function in equation (16) and the wealth transformation function in (15), the expected value of the marginal utility of the end of period wealth is

$$
\begin{aligned}
E\left[U^{\prime}\left(W_{T}\right)\right] & =E\left[\exp \left(\gamma h_{W_{T}}\left(W_{T}\right)\right)\right] \\
& =E\left[\exp \left(\gamma \mu_{W_{T}}+\gamma \sigma_{W_{T}} y\right)\right] \\
& =e^{\gamma \mu_{W_{T}}} \int_{0}^{\infty} \frac{a^{p+q}}{\Gamma(p+q)} y^{p+q-1} e^{-\left(a-\gamma \sigma_{W_{T}}\right) y} d y
\end{aligned}
$$

Changing variables

$$
E\left[U^{\prime}\left(W_{T}\right)\right]=\frac{a^{p+q} e^{\gamma \mu_{W_{T}}}}{\left(a-\gamma \sigma_{W_{T}}\right)^{p+q}} \frac{1}{\Gamma(p+q)} \int_{0}^{\infty} z^{p+q-1} e^{-z} d z
$$

Since the integral on the RHS is equal to the gamma function $\Gamma(p+q)$, the expected value of the marginal utility of wealth is

$$
E\left[U^{\prime}\left(W_{T}\right)\right]=\frac{a^{p+q} e^{\gamma \mu_{W_{T}}}}{\left(a-\gamma \sigma_{W_{T}}\right)^{p+q}} .
$$

Substituting equations (16) and (37) into equation (4) and simplifying yields equation (17).

Proof. (Equation 18 on the asset specific pricing kernel) This proof is presented in three steps. First, the conditional density of $W_{T}$ given $S_{T}$ is obtained. Second, this result is applied to obtain $E\left[U^{\prime}\left(W_{T}\right) \mid S_{T}\right]$. Finally, we obtain the asset specific pricing kernel. 
The conditional density of $y$ given $x$ is

$$
\begin{aligned}
f(y \mid x) & =\frac{f(y, x)}{f(x)} \\
& =\frac{a^{q}}{\Gamma(q)}(y-x)^{q-1} e^{-a(y-x)}
\end{aligned}
$$

where the second equality follows directly from Definition 1 . The conditional density of $W_{T}$ given $S_{T}$ is readily available by the transformations in Definition 2. This concludes the first part of the proof.

The expected value of the marginal utility of the end of period wealth conditioned to the terminal value of the underlying is

$$
\begin{aligned}
E\left[U^{\prime}\left(W_{T}\right) \mid S_{T}\right] & =E\left\{\exp \left[\gamma h_{W_{T}}\left(W_{T}\right)\right] \mid S_{T}\right\} \\
& =\int \exp \left[\gamma h_{W_{T}}\left(W_{T}\right)\right] f\left(W_{T} \mid S_{T}\right) d W_{T} .
\end{aligned}
$$

Changing variables and after some tedious algebra we obtain

$$
E\left[U^{\prime}\left(W_{T}\right) \mid S_{T}\right]=\frac{a^{q} \exp \left[\gamma \mu_{W_{T}}+\gamma \sigma_{W_{T}}\left(\frac{h\left(S_{T}\right)-\mu}{\sigma}\right)\right]}{\left(a-\gamma \sigma_{W_{T}}\right)^{q}} \frac{1}{\Gamma(q)} \int_{0}^{\infty} z^{q-1} e^{-z} d z
$$

Since the integral on the RHS is equal to gamma function $\Gamma(q)$,

$$
E\left[U^{\prime}\left(W_{T}\right) \mid S_{T}\right]=\frac{a^{q} \exp \left[\gamma \mu_{W_{T}}+\gamma \sigma_{W_{T}}\left(\frac{h\left(S_{T}\right)-\mu}{\sigma}\right)\right]}{\left(a-\gamma \sigma_{W_{T}}\right)^{q}},
$$

which completes the second part of the proof.

Finally, substituting equations (37) and (38) into equation (5) and simplifying yields equation (18).

Proof. (Equation 23 on the log gamma equilibrium relationship) Using equations 
(14), (18) and (6) yields

$$
\begin{aligned}
F= & E\left(\psi S_{T}\right) \\
= & \int_{h^{-1}(\mu)}^{h^{-1}(\infty)} S_{T} \frac{\left(a-\gamma \sigma_{W_{T}}\right)^{p}}{\Gamma(p)} \frac{h^{\prime}\left(S_{T}\right)}{\sigma}\left(\frac{h\left(S_{T}\right)-\mu}{\sigma}\right)^{p-1} \\
& \exp \left[-\left(a-\gamma \sigma_{W_{T}}\right) \frac{h\left(S_{T}\right)-\mu}{\sigma}\right] d S_{T} .
\end{aligned}
$$

Changing variables, simplifying and recalling that $h\left(S_{T}\right)=\ln \left(S_{T}\right)$ yields,

$$
F=e^{\mu} \frac{\left(a-\gamma \sigma_{W_{T}}\right)^{p}}{\left(a-\gamma \sigma_{W_{T}}-\sigma\right)^{p}} \int_{0}^{\infty} \frac{z^{p-1}}{\Gamma(p)} e^{-z} d z .
$$

Using the definition of a gamma distribution, the integral on the RHS equals one. Therefore,

$$
F=e^{\mu} \frac{\left(a-\gamma \sigma_{W_{T}}\right)^{p}}{\left(a-\gamma \sigma_{W_{T}}-\sigma\right)^{p}}
$$

Rearranging the terms yields equation (23)

Proof. (Equation 24 on the log gamma option pricing formula) The call option pricing formula is given by

$$
\begin{aligned}
C e^{r T}= & E\left[\max \left(S_{T}-K, 0\right) \psi\right] \\
= & \int_{h^{-1}(\mu)}^{h^{-1}(\infty)} \max \left(S_{T}-K, 0\right) \frac{\left(a-\gamma \sigma_{W_{T}}\right)^{p}}{\Gamma(p)} \frac{h^{\prime}\left(S_{T}\right)}{\sigma} \\
& \left(\frac{h\left(S_{T}\right)-\mu}{\sigma}\right)^{p-1} \exp \left[-\left(a-\gamma \sigma_{W_{T}}\right) \frac{h\left(S_{T}\right)-\mu}{\sigma}\right] d S_{T} .
\end{aligned}
$$

Using the fact that $P(x>X)=1-P(x \leq X)$, changing variables and noting 
that $h\left(S_{T}\right)=\ln \left(S_{T}\right)$, returns.

$$
\begin{aligned}
C e^{r T}= & \int_{0}^{\infty}\left(e^{\mu+\sigma x}-K\right) \frac{\left(a-\gamma \sigma_{W_{T}}\right)^{p}}{\Gamma(p)} x^{p-1} e^{-\left(a-\gamma \sigma_{W_{T}}\right) x} d x \\
& -\int_{0}^{\frac{\ln (K)-\mu}{\sigma}}\left(e^{\mu+\sigma x}-K\right) \frac{\left(a-\gamma \sigma_{W_{T}}\right)^{p}}{\Gamma(p)} x^{p-1} e^{-\left(a-\gamma \sigma_{W_{T}}\right) x} d x
\end{aligned}
$$

Expanding the integrals, changing variables again and using equation (23), yields the option pricing formula given in equation (24). 


\section{References}

[1] Arrow, K. "The Theory of Risk Aversion." In: Essays in the theory of riskbearing, North-Holland Publishing Co. (1974), p.90-120.

[2] Black F., and M. Scholes. "The Pricing of Options and Corporate Liabilities." Journal of Political Economy, 81 (1973), 637-654.

[3] Brennan, M. "The Pricing of Contingent Claims in Discrete Time Models." Journal of Finance, 34 (1979), 53-68.

[4] Camara, A. "A Generalization of the Brennan-Rubinstein Approach for the Pricing of Derivatives." Journal of Finance, 58 (2003), 805-821.

[5] Franke, G., and J. Huang, and R. Stapleton. "Two-Dimensional RiskNeutral Valuation Relationships For the Pricing of Options." Working Paper, Manchester University (2004).

[6] Gerber, H., and E. Shiu. "Otion Pricing by Esscher Transforms." Transactions of Society of Actuaries, 46 (1994), 99-140.

[7] Heston, S. "Invisible Parameters in Option Prices." Journal of Finance 48 (1993), 933-947.

[8] Huang C., and R. Litzenberger. Foundations for Financial Economics. New York, USA: North-Holland Publishing Co. (1988).

[9] Hutchinson, T., and C. Lai Continuous Bivariate Distributions, Emphasising applications. Rumsby Scientific Publishing (1990). 
[10] Lane, M., and O. Movchan. "The Perfume of a Premium II." Derivatives Quarterly, 5 (1999), 27-40.

[11] Loukas, A., and L. Vasiliades, and N. Dalezios, and C. Domenikiotis. "Rainfallfrequency Mapping for Greece." Physics and Chemistry of the Earth, Part B: Hydrology, Oceans and Atmosphere, 26 (2001), 669-674.

[12] Mardia, K. V. Families of Bivariate Distributions. Griffin (1970).

[13] McKay, A. T. "Sampling from Batches." Journal of the Royal Statistical Society, series B, 1 (1934), 207-216.

[14] Poon, S., and R. Stapleton. Asset Pricing Theory: a Discrete Time, Complete Markets Approach. Oxford University Press (2005).

[15] Rubinstein, M. "The Valuation of Uncertain Income Streams and the Pricing of Options." Bell Journal of Economics and Management Science, 7 (1976), 407-425.

[16] Savickas, R. "A Simple Option Pricing Formula." Financial Review, 37 (2002), 207-226.

[17] Schroder, M. "Risk-Neutral Parameters Shifts and Derivatives Pricing in Discrete Time." Journal of Finance, 59 (2004), 2375-2401.

[18] Sharda, V., and P. Das. "Modelling Weekly Rainfall Data for Crop Planning in a Sub-Humid Climate of India." Agricultural Water Management, 76 (2005), 120-138.

[19] Stern, R., and R. Coe. "A Model Fitting Analysis of Daily Rainfall Data." Journal of the Royal Statistical Society, series A, 147 (1984), 1-34. 
[20] Yue, S. and T. Ourada, and B. Bobee. "A Review of Bivariate Gamma Distribution for Hydrological Application." Journal of Hydrology, 246 (2001), 1-18. 